\title{
Is treatment for bipolar disorder more effective earlier in illness course? A comprehensive literature review
}

\author{
Katie Joyce ${ }^{1}$, Andrew Thompson ${ }^{2,3}$ and Steven Marwaha $a^{2,4^{*}}$ [0
}

\begin{abstract}
Background: We aimed to investigate a key element of the early intervention approach; whether treatment at an earlier stage of bipolar disorder is more effective than later in its course.

Methods: A comprehensive literature review using Medline, Embase, Psychinfo, PsycArticle and Web of Science as data sources, with a subsequent narrative synthesis. Study quality was assessed using the Cochrane risk of bias method.

Results: Our search strategy yielded eight primary papers and two meta-analyses (of psychological therapies and Olanzapine) in total representing 8942 patients. Five studies focused on comparisons between first and multiple episode; the others on fewer vs more episode categories. There was a consistent finding suggesting treatment in earlier illness stage resulted in better outcomes in terms of response, relapse rate, time to recurrence, symptomatic recovery, remission, psychosocial functioning and employment. This effect was found for pharmacological (Lithium, Olanzapine, Divalproex) and psychological treatment.

Limitations: There was high risk of selection, performance and attrition bias in most studies. First admission or presentation is unlikely to equate to first episode because of duration of untreated illness. Some patients having experienced multiple episodes could be "treatment resistant". Study heterogeneity precluded meta-analysis.

Conclusions: Psychological and pharmacological treatment in the early stages of illness is more effective than in later stages of bipolar disorder across multiple domains. There is a first episode and early phase effect. Consistent with the staging model of illness findings provide evidence for the clinical utility of an early intervention approach in bipolar disorder to improve patient outcomes.
\end{abstract}

Keywords: Bipolar disorder, Early intervention, First episode, Effectiveness, Treatment, Multiple episodes

\section{Background}

Mental illness causes considerable global disease burden, often affecting patients for their entire lifespan (Whiteford et al. 2013). Bipolar disorder, including subthreshold disorders, affect approximately $2 \%$ of the population (Merikangas et al. 2011). It ranks as a major global cause of disability adjusted life years for 10-24 year olds (Gore et al. 2011), and people with the disorder are seven times

\footnotetext{
*Correspondence: s.marwaha@warwick.ac.uk

${ }^{2}$ Unit of Mental Health and Wellbeing, Division of Health Sciences, Warwick Medical School, University of Warwick, Coventry CV4 7AL, UK Full list of author information is available at the end of the article
}

more likely to die an unnatural death (Hayes et al. 2015). As well as the significant impact on public health, costs of the disorder are very high (Pari et al. 2014), in part because of the functional losses associated with increasing episode number (Marwaha et al. 2013).

Whilst many expert researchers (Berk et al. 2009; Martin et al. 2013; Salvadore et al. 2008) have suggested early intervention (EI) could reduce the cumulative morbidity, early death and financial costs of bipolar disorder, for this case to be persuasive several pieces of evidence need to align. Failure to make contact with mental health services and receive a prompt correct diagnosis 
and initiation of treatment appear to be a problem for a range of mental disorders (Wang et al. 2005), but this appears particularly true for bipolar disorder. There are high levels of unmet need in young people with bipolar disorder (Charney et al. 2003). Delays in diagnosis are lengthy (mean 12.5 years), clinically important, and associated with social dysfunction (Matza et al. 2005), as well as an increased risk of lifetime suicidality (Hawton et al. 2005; Nery-Fernandes et al. 2012). Indeed, the delay in diagnosis risks young people receiving inappropriate treatment, which may worsen the condition. Thus, the early diagnosis and early initiation of treatment appear important in bipolar disorder and this would support an EI approach.

If research identifies treatment is most effective earlier in illness course or soon after first episode, then a second and critical evidential strand would support the rationale for EI in bipolar disorder. This would clearly demonstrate that outcomes could be changed by having a focus early in the course of the disorder, thus moulding clinical treatment priorities. Such evidence is likely to support the further development and implementation of EI services for people with bipolar disorder, consistent with the clinical staging approach to managing mental disorders (McGorry 2007).

Therefore, we aimed to review the extant literature, to investigate whether treatment at an earlier stage of bipolar (e.g., after the first episode) is more effective than later in the course of the disorder.

\section{Methods}

A comprehensive review of the literature was completed. An initial scoping review enabled search terms to be selected and refined. This also allowed the development of predetermined inclusion and exclusion criteria for article selection to improve search strategy and to reduce bias in paper selection.

\section{Sources of information}

Using a systematic process, an extensive search of papers catalogued in Medline, Embase, Psychinfo, Psycarticle, and Web of Science was carried out in October 2015. Papers in the English language were searched, with no criterion for publication date. Following this, all abstracts were downloaded into the Endnote referencing system and duplicates were deleted. The inclusion and exclusion criteria were applied to each abstract to create a list of papers for full-text retrieval. The reference lists of the included papers were examined for further papers, and the authors of the most relevant studies were contacted requesting details of any other studies which they thought were important in the area.

\section{Search terms}

The search terms were arranged in groups and included mesh terms, (group 1); bipolar, or mania, or hypomania, or manic depression, AND (group 2); the early treatment, or treatment onset, or the early intervention, or first episode, or multiple episode, or incident, AND (group 3); outcome, or recovery, or relapse, or recurrence, or remission, NOT (group 4); and genetics, or surgery, or dementia, or diabetes, or elderly or cardiovascular disease.

\section{Inclusion and exclusion criteria}

Inclusion criteria were: (a) patients diagnosed with bipolar disorder (any type), (b) the direct comparison of outcome in the same study between treatment received earlier (including at first episode) with later in illness course. We also included studies which had sampled hospital inpatients and compared those earlier vs later in illness course. Exclusion criteria were: (a) non-English language papers, (b) studies in which the only population sampled were children, and (c) case-series data.

\section{Data extraction}

The full text of all included studies was scrutinised and a narrative synthesis completed directed at answering the research aims. Data were extracted on sample size, sampling frame, study design, outcomes, and main results.

\section{Results}

The search generated 1311 abstracts, and after applying the inclusion and exclusion criteria, the full text of 17 papers was retrieved. Seven full-text papers were excluded. The main reasons for study exclusion subsequent to full-text retrieval were either a lack of comparison with earlier or first episode $(N=3)$, or no comparison of treatment between groups $(N=2)$. On contacting relevant authors, one author replied with three study suggestions, one of which was already included and two that did not fulfil inclusion criteria. After reviewing the full-text studies, eight primary studies and two reviews were included for the final review. A PRISMA flowchart (Fig. 1) illustrates the number of studies included and excluded at each stage of the process. Table 1 shows the study characteristics and main findings. The total number of patients in reviewed studies was 8942 . We do not present a meta-analytic synthesis of the studies due to heterogeneity and instead structure our findings narratively, reviewing psychological or pharmacological treatment and inpatient studies.

Study quality was appraised using the Cochrane risk of bias assessment (Higgins et al. 2008) and an overall view of the quality of the literature can be seen in Fig. 2. There was a high risk of selection, performance, and attrition 


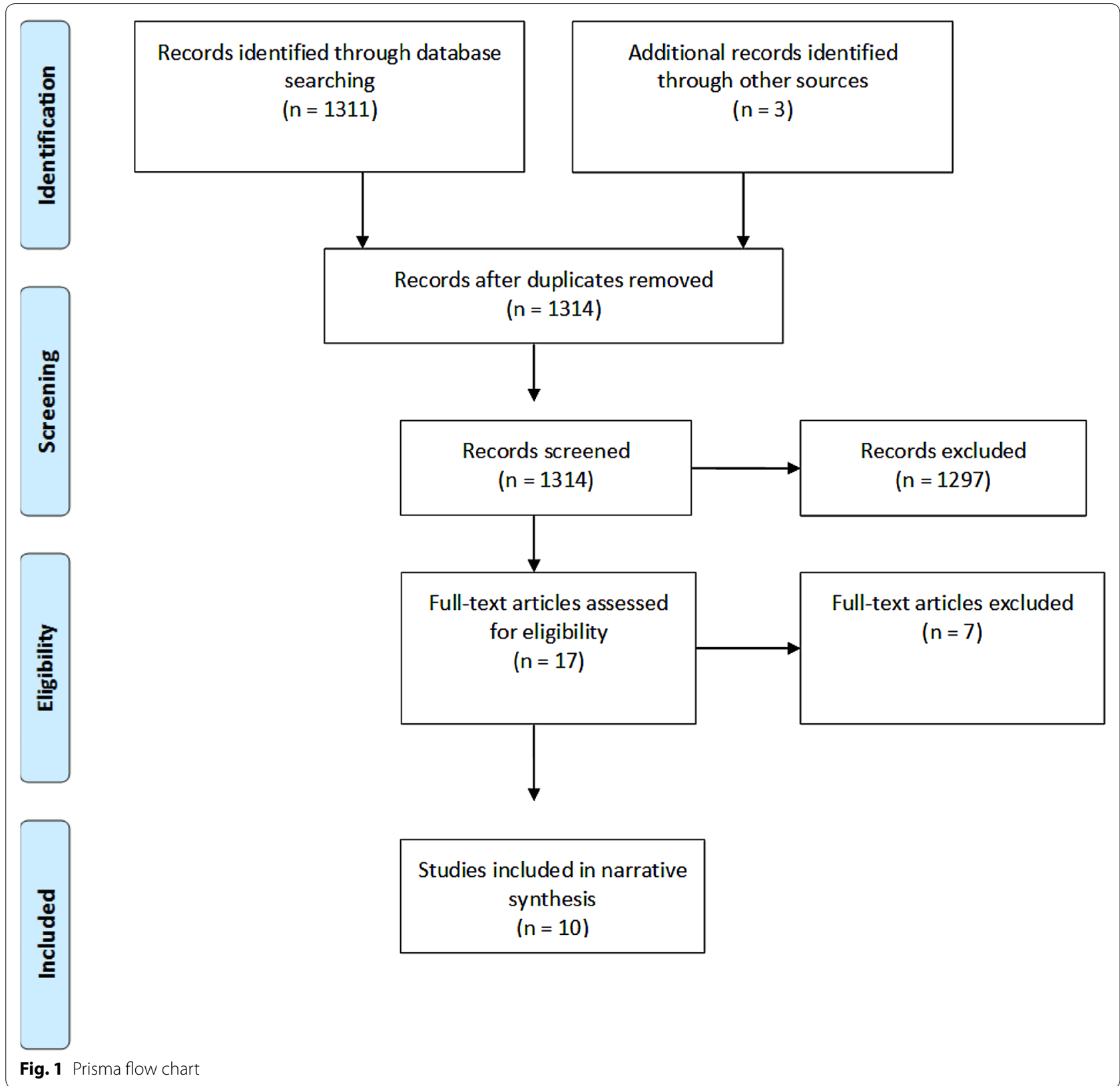

bias in most studies. However, in the main, studies demonstrated a low risk of reporting bias, providing results for all outcomes that they measured.

\section{Studies of psychological treatment}

Colom et al. (2010) investigated whether treatment response is affected by course of illness progression using the Kaplan-Meier survival analysis. They conducted a post-hoc sub analysis on 120 euthymic bipolar patients, assessing time to recurrence in patients undergoing group psychoeducation in relation to course of their illness. Patients who had experienced six episodes or less demonstrated a reduced time to recurrence $(p=0.038)$ from the addition of psychoeducation to treatment. Patients who had experienced more than seven episodes showed no significant difference in outcome by the addition of psychoeducation. In considering time spent ill, those who had experienced less than six episodes showed a reduction in time spent in any episode polarity with the psychoeducation treatment.

A meta-analysis of the effectiveness of psychological therapies as adjuncts to usual pharmacotherapy was 


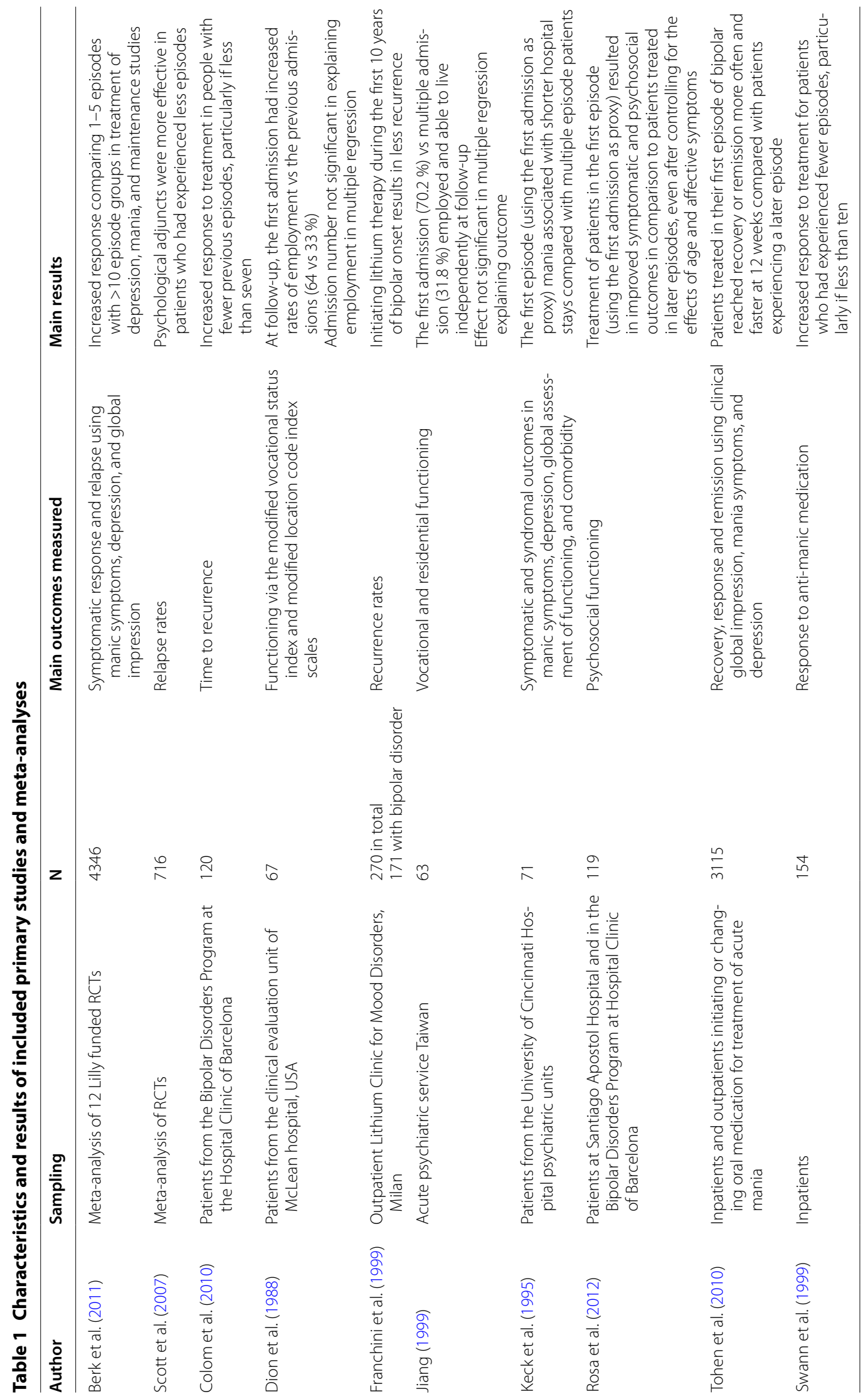




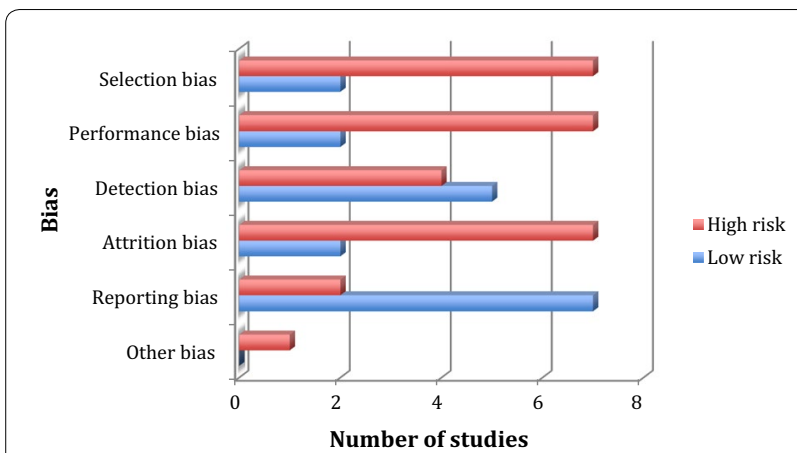

Fig. 2 Bar chart to show risk of bias in included studies

completed by Scott et al. (2007). Data were synthesized from nine studies examining cognitive behaviour therapy, interpersonal social rhythms therapy, family focused therapy, and psychoeducation. Again, they found that adjuncts to treatment were less efficacious in preventing relapse if the patient had experienced more episodes. A significance difference emerged between relapse rates in patients who had experienced twelve episodes or less.

\section{Studies of pharmacological treatment}

Tohen et al. (2010) conducted a two-year prospective observational study, comparing the efficacy of oral medication given after the first episode of mania with oral medication given after multiple episodes. The sample size was large $(N=3115)$. They also investigated the different characteristics of first episode and multiple episode bipolar patients, assessing patients using the Young Mania Rating Scale (YMRS), Clinical Global Impressions-Bipolar Disorder (CGI-BP) mania scores, CGI-BP depression, and the Hamilton Depression Rating Scale. Cox proportional hazard modelling was used as the method of analysis. During the study period, patients treated in their first episode not only reached recovery (anytime during study 37.9 vs $32.0 \%$, end of 12 weeks 39.6 vs $33.1 \%$ ) or remission (first vs multiple episode 89.0 vs $81.4 \%$ ) more often, but they did so significantly more rapidly than patients treated in their later episode $(p=0.0126)$. Notably, this was despite patients in their first episode having higher mania ratings (YMRS) than multiple episode patients at baseline. It should be noted that some patients entered into this study were currently medicated (but requiring a change) and the findings refer to episode number as opposed to the duration of illness.

Using a double blind RCT design, Swann et al. (1999) investigated the effect of episode number on response to treatment in 154 inpatients with bipolar disorder. Participants were randomly assigned to Divalproex, Lithium, or placebo medication. To investigate the effect of illness course on response to treatment, the number of the previous episodes was compared using improvement in the manic syndrome score from the Schedule for Affective Disorders and Schizophrenia (SADS) as the main outcome. An improved response to anti-manic medication was evident as measured by the SADS manic syndrome score in patients who had experienced fewer episodes. The pivotal point by which intervention must have been initiated to improve treatment response was approximately ten episodes.

These results are similar to the another investigation of the effect of illness stage on response to lithium prophylaxis. Franchini et al. (1999) sampled a mixed group of patients with mood disorders $(N=270)$, but this included 171 people with bipolar disorder. They found that the earlier commencement of lithium prophylaxis (within the first 10 years of bipolar onset) was associated with improved treatment outcome as measured by recurrence rate of an affective episode even after adjustment for polarity, sex, age, onset, duration of illness, and duration of lithium prophylaxis. Furthermore, the significant association between treatment onset and outcome was stronger for 'very early' and 'early' lithium onset in comparison to the Lithium initiation after 11 years of preceding illness. The results did not vary between those with recurrent depressive disorder and people with bipolar disorder and polarity of the illness did not mediate the impact of lithium on patient outcome.

A meta-analysis (Berk et al. 2011) reviewed 12 randomised control studies to examine the effect of illness staging on outcome in bipolar disorder with regard to treatment response with Olanzapine. Symptomatic response rates to treatment, as defined by $>50 \%$ reduction from baseline, were better in individuals who had experienced less than five episodes with response rate worsening after this point. There was a significant difference in the rates of response for those studies examining mania (52-69 \% for $<5$ episodes vs $29-59 \%$ for $>5$ episodes). On most outcome measures, the odds of responding were increased by a factor of two in those who had experienced less than five episodes.

The effect was less clear-cut for depression studies, in which rates of response were significantly higher for the 1-5 episode group on only two measures. In terms of studies investigating maintenance treatment, relapse was reduced by $40-60 \%$ for those who had experienced $1-5$ episodes or 6-10 episodes compared with the $>10$ episode group with this being statistically significant with regard to relapse to mania only $(p=0.005)$.

\section{Studies of hospital inpatient treatment}

A number of hospital inpatient studies were identified as providing suitable data. Whilst these studies were not comparing a single treatment between groups, we 
equated hospital admission with hospital treatment. Whether the treatment was pharmacological and/or psychological was not explicitly stated, but is likely to have involved both.

Sampling patients with bipolar disorder, Keck et al. (1995) report that patients with multiple episodes $(N=37)$ required longer hospital stays to achieve recovery in comparison to people with first episodes $(N=34)$. Furthermore, the increased requirement for treatment was despite similar ratings of illness severity on a variety of measures, including the YMRS, Hamilton Rating Scale for Depression, the Scale for Assessment of Positive Symptoms, and the Global Assessment Scale. The main limitation to the study was one of small sample sizes, though it is noteworthy that the participants were patients with severe symptoms by virtue of the sampling strategy.

Rosa et al. (2012) used a naturalistic design to investigate the differences in psychosocial functioning in 119 hospitalized bipolar patients comparing those experiencing their first episode and multiple episodes. Significantly, more patients in the first-episode group had symptomatic recovery (vs multiple episodes) at 12 months (62.7 vs $44.9 \%, p=0.005)$. Patients being treated in their first episode had better functional outcomes at 6 and 12 months compared with those being treated in later episodes in diverse outcomes, such as autonomy, occupation, cognition, interpersonal relationships, and leisure time even after controlling for the effects of age and affective symptoms at 12 months (all $p<0.005$ ).

Dion et al. (1988) investigated the relationship between symptoms and functioning in patients with bipolar disor$\operatorname{der}(N=67)$ admitted to hospital at a 6-month follow-up point. Comparing the first admission patients with those with one or more previous admissions, they found that the first admission group had a higher level of independent living at follow-up (85 vs $66 \%$ ). In terms of employment, the first admission group also fared better (66 vs $33 \%$ ) assessed by the vocational status index, but in a multiple regression analysis, admission number did not predict employment status at 6 months.

Jiang (1999) conducted a prospective study investigating functional outcomes in 63 bipolar disorder patients who were admitted to hospital in Taiwan. In comparison to those who had experienced multiple admissions, the first admission group showed significantly higher vocational functioning $(p=0.034)$ and were more often employed (70 vs $31.8 \%$ ). Whilst more of the first admission group were living independently (70 vs $40 \%$ ) at year 1 , this was not statistically significant $(p=0.24)$. However, the number of admissions was not an independent predictor of vocational functioning after controlling for confounders using a regression analysis.

\section{Discussion}

\section{Main findings}

A comprehensive review of the current literature was completed to investigate whether the effectiveness of treatment of bipolar disorder varies depending on the illness stage. In summary, the literature suggests that treatment earlier in the course of illness is more effective than in the later stages of bipolar disorder. Whilst being based on a small number of studies, this finding is seen for both psychological and pharmacological therapies and the effect is apparent in a range of functional, symptomatic, recurrence, and relapse outcome measures. When confounders were controlled, this effect was attenuated and rendered non-significant in two studies.

\section{Limitations of the literature}

There were several methodological limitations to the literature that frames our findings. We wished to answer the question of whether treatment is more effective earlier in illness course. A suitable methodologically robust study design would be to sample treatment naïve individuals with a first episode and multiple previous episodes of illness and compare treatment effectiveness between the groups. We did not identify any studies using this methodology.

A related point is that many of the identified studies included those with multiple episodes who had already received treatment within the previous episodes. A proportion of these patients may have been "treatment resistant", defined as having received two consecutive medications without recovery (Gitlin 2006). That group may have by definition, been less likely to respond to treatment in comparison to those with first episode and this could bias results in favour of treatment effectiveness earlier in illness course. In the main, it is unknown what proportion of the populations in these studies could be categorised as treatment resistant. However, in one study, approximately $50 \%$ of the sample had previously found the treatment either ineffective or were intolerant to it (Bowden et al. 1994; Swann et al. 1999).

Studies from which relevant data could be extracted showed substantial variations in study design, sampling frames, analytic strategies, and outcomes measured. In addition, whilst nine studies used episode number as the category for comparison, one study reported length of illness (e.g., less than 10 years). Because of this heterogeneity in the literature, we did not statistically combine results in a meta-analysis, as in our judgement, this not feasible or considered potentially meaningful. Instead, we completed a narrative synthesis as the most methodologically sound way to understand the underlying patterns in the literature given the limitations described above. This type of synthesis enabled a richer understanding of the extant literature. 
There may have been clinical and socio-demographic differences between the first and multiple episode groups, which could have had independent effects on outcome (Berk et al. 2011; Tohen et al. 2010) whether these were measured or not. Confounders, such as age, have been controlled for in some analyses and significant differences remain between the groups (Franchini et al. 1999; Rosa et al. 2012). In other studies, despite striking absolute differences in outcome (e.g., employment) between the first and multiple admission groups, admission number did not remain significant after controlling for other factors (Jiang 1999). Variable adjustment for confounding and disparate analytic strategies (due to the nature of studies included) means that caution is needed in direct comparisons between studies.

How first episode was classified frequently relied on a first admission to hospital with four studies using the first admission as a proxy for first episode. This is important given that prior to clinical diagnosis, patients have often experienced the previous affective symptoms (Martin et al. 2013) and duration of untreated illness can be lengthy (Berk et al. 2007; Murru et al. 2015). This is the case even after presentation to mental health services (Patel et al. 2015). There is a danger then in reviewing the literature that first episode status is conflated with the first admission or first contact with medical services, when, in fact, this is not the case. This potential variation between studies means that caution should be exercised in conclusions, regarding episode number and treatment effectiveness. It is also difficult to make direct comparison between the bulks of studies that investigate the impact of episode number on treatment effectiveness with a study that details length of illness (Franchini et al. 1999) as the variable analysed.

There were only five studies that compared people with first episode bipolar (with four relying on the first admission for this categorisation) and those with further episodes. Therefore, the current available literature (which included two meta-analyses) is weighted towards the comparisons of people who have already experienced a number of episodes with those having experienced more.

Finally, there may be a treatment confounding effect apparent in our results. For example, patients with the first episode bipolar disorder or in the early stages may have received more robust care than patients in comparison groups given the high priority now given globally to first episode mental disorders. This, of course, is less likely to be an issue for older studies.

\section{Effectiveness of treatments in multiple domains}

Psychological or pharmacological treatments at an earlier stage of bipolar disorder are more effective that in the later stages. This is apparent in multiple domains covering outcomes of importance to both clinicians and patients. The literature spans greater effectiveness on relapse, remission, recovery rates, comorbidity, symptomatic and syndromal outcome, global psychosocial functioning, and vocational and residential functioning. The fact that the same trend is seen with different treatment modalities, as well as a variety of outcomes adds validity and potency to our findings.

\section{Timing}

The evidence base in the first episode psychosis suggests that using a stage-specific approach to treatment in first episode of illness is more effective than not (Marshall et al. 2011). The underlying tenet of this approach was supported by the current literature review. However, a number of studies, including the two meta-analyses, suggest even after a first episode, less episode number is associated with greater treatment effectiveness. This finding was independent of treatment studied (Lithium, Olanzapine, Divalproex, CBT, psychoeducation) or study design (Berk et al. 2011; Colom et al. 2010; Scott et al. 2007; Swann et al. 1999).

The pivotal point at which earlier treatment was more effective ranged from 5 to 12 episodes. Given the differences between studies and the limitations of the literature, it is difficult to be sure that a pivotal episode number exists between this range after which effectiveness changes or whether this is simply a function of how data were categorised and analysed. Further research is necessary to definitively answer this question.

The early treatment would seem important, but our findings suggest an early phase effect as opposed to a solely first episode effect. A possible interpretation is that for some people, it takes a number of episodes to achieve medication optimisation and adequate adherence and to be able to engage fully in therapy. Whilst the early intervention services for bipolar disorder and their evaluation are in their infancy (Marwaha et al. 2016), evidence from an RCT sampling people early in illness course does suggest that specialised and systematic treatment is more clinically and cost-effective than the standard outpatient care (Kessing et al. 2013). The findings of this review would support an extension of this approach.

\section{Why does treatment earlier in illness course improve outcomes?}

The clinical staging approach for bipolar disorder suggests a model, in which there is a progression from "at risk" symptoms to the first presentation, to multiple episodes right through to refractory illness (Kapczinski et al. 2009). Movement through the stages can be due to a combination of genetic vulnerability, life stresses, and substance misuse, and each stage may be linked to 
abnormalities in biomarkers, such as TNF-alpha, BDNF, and 3-nytrotyrosine (Kauer-Sant'Anna et al. 2009). Advancing illness stage is associated with neuroprogression evidenced by the changes in the brain structure (especially in the fronto-limbic system) (Berk et al. 2011; Mwangi et al. 2016). Alongside these biological changes, there is evidence for a progressively smaller inter-episode period, as episode number increases. Whilst the earlier episodes may need to be triggered, the illness progresses episodes can begin to emerge spontaneously. This has been conceptualized into a stress sensitization-kindling model of bipolar disorder, in which repeated abnormal brain activity reduces the threshold for repeat events increasing the risk of relapse (Post 2007).

These factors are very likely to form part of the explanation for our findings that treatment early in illness course is more effective than in later episodes in terms of both clinical and symptomatic outcomes. This review suggests that the progression to later stages of illness is associated with treatments becoming less effective and these chimes with the requirement for more complex treatment regimes for many people who have well-established bipolar disorder (Post et al. 2010). The greater effectiveness in improving functional outcomes in the early course may be particularly linked to the initial appearance and worsening of cognitive impairments with time, a factor which is known to independently predict vocational functioning in bipolar disorder (Gilbert et al. 2013; Torres et al. 2011). Our findings also paradoxically highlight the scale of the therapeutic challenge to assist people in later stages of the illness, in which there appears to be some level of treatment resistance.

To conclude, this literature review found substantial evidence that both pharmacological and psychological treatments for bipolar disorder are more effective in the earlier stages of illness. The effect, which is demonstrable at the first episode, is also apparent in the early phases of treatment. The findings provide some evidence for the clinical and policy rationale of an early intervention approach in bipolar disorder to improve patient outcomes.

\section{Abbreviations \\ El: early intervention; YMRS: Young Mania Rating Scale; CGI-BP: Clinical Global Impressions-Bipolar Disorder; SADS: Schedule for Affective Disorders and Schizophrenia. \\ Authors' contributions \\ $\mathrm{KJ}$ developed study procedures, conducted the search, reviewed papers, and wrote the initial drafts of the manuscript. AT substantially contributed to the analysis and writing of the manuscript. SM conceived of the study, oversaw all aspects, and wrote drafts of the manuscript. All authors read and approved the final manuscript.}

\section{Author details}

${ }^{1}$ Warwick Medical School, University of Warwick, Coventry CV4 7AL, UK. ${ }^{2}$ Unit of Mental Health and Wellbeing, Division of Health Sciences, Warwick Medical
School, University of Warwick, Coventry CV4 7AL, UK. ${ }^{3}$ North Warwickshire Early Intervention in Psychosis Service, Coventry and Warwickshire NHS Partnership Trust, Coventry, UK. ${ }^{4}$ Affective Disorders Service (IPU 3-8), Caludon Centre, Coventry, UK.

\section{Competing interests}

The authors declare that they have no competing interests.

Received: 22 June 2016 Accepted: 30 August 2016

Published online: 09 September 2016

\section{References}

Berk M, Brnabic A, Dodd S, Kelin K, Tohen M, Malhi GS, et al. Does stage of illness impact treatment response in bipolar disorder? Empirical treatment data and their implication for the staging model and early intervention. Bipolar Disord. 2011a;13(1):87-98.

Berk M, Dodd S, Callaly P, Berk L, Fitzgerald P, De Castella A, et al. History of illness prior to a diagnosis of bipolar disorder or schizoaffective disorder. J Affect Disord. 2007;103(1):181-6.

Berk M, Kapczinski F, Andreazza A, Dean O, Giorlando F, Maes M, et al. Pathways underlying neuroprogression in bipolar disorder: focus on inflammation, oxidative stress and neurotrophic factors. Neurosci Biobehav Rev. 2011b;35(3):804-17.

Berk M, Malhi GS, Hallam K, Gama CS, Dodd S, Andreazza AC, et al. Early intervention in bipolar disorders: clinical, biochemical and neuroimaging imperatives. J Affect Disord. 2009;114(1):1-13.

Bowden CL, Brugger AM, Swann AC, Calabrese JR, Janicak PG, Petty F, et al. Efficacy of divalproex vs lithium and placebo in the treatment of mania. JAMA. 1994:271(12):918-24.

Charney DS, Reynolds CF, Lewis L, Lebowitz BD, Sunderland T, Alexopoulos GS, et al. Depression and Bipolar Support Alliance consensus statement on the unmet needs in diagnosis and treatment of mood disorders in late life. Arch Gen Psychiatry. 2003;60(7):664-72.

Colom F, Reinares M, Pacchiarotti I, Popovic D, Mazzarini L, Martínez-Arán A, et al. Has number of previous episodes any effect on response to group psychoeducation in bipolar patients? A 5-year follow-up post hoc analysis. Acta Neuropsychiatr. 2010;22(2):50-3.

Dion GL, Tohen M, Anthony WA, Waternaux CS. Symptoms and functioning of patients with bipolar disorder six months after hospitalization. Hosp Community Psychiatry. 1988;39(6):652-7.

Franchini L, Zanardi R, Smeraldi E, Gasperini M. Early onset of lithium prophylaxis as a predictor of good long-term outcome. Eur Arch Psychiatry Clin Neurosci. 1999;249(5):227-30.

Gilbert E, Marwaha S. Predictors of employment in bipolar disorder: a systematic review. J Affect Disord. 2013;145(2):156-64.

Gitlin M. Treatment-resistant bipolar disorder. Mol Psychiatry. 2006;11(3):227-40.

Gore FM, Bloem PJ, Patton GC, Ferguson J, Joseph V, Coffey C, et al. Global burden of disease in young people aged 10-24 years: a systematic analysis. Lancet. 2011;377(9783):2093-102.

Hawton K, Sutton L, Haw C, Sinclair J, Harriss L. Suicide and attempted suicide in bipolar disorder: a systematic review of risk factors. J Clin Psychiatry. 2005;66:693-704.

Hayes J, Miles J, Walters K, King M, Osborn D. A systematic review and metaanalysis of premature mortality in bipolar affective disorder. Acta Psychiatr Scand. 2015;131(6):417-25.

Higgins JP, Green S. Cochrane handbook for systematic reviews of interventions. Chichester: Wiley; 2008.

Jiang HK. A prospective one-year follow-up study of patients with bipolar affective disorder. Zhonghua Yi Xue Za Zhi. 1999;62(8):477-86 (Free China ed.).

Kapczinski F, Dias WV, Kauer-Sant'Anna M, Frey BN, Grassi-Oliveira R, Colom F, et al. Clinical implications of a staging model for bipolar disorders. Expert Rev Neurother. 2009:9(7):957-66.

Kauer-Sant'Anna M, Kapczinski F, Andreazza AC, Bond DJ, Lam RW, Young LT, et al. Brain-derived neurotrophic factor and inflammatory markers in patients with early-vs late-stage bipolar disorder. Int J Neuropsychopharmacol. 2009:12(4):447-58. 
Keck PE Jr, McElroy SL, Strakowski SM, West SA, Hawkins JM, Huber TJ, et al. Outcome and comorbidity in first-compared with multiple-episode mania. J Nerv Ment Dis. 1995;183(5):320-4.

Kessing LV, Hansen HV, Hvenegaard A, Christensen EM, Dam H, Gluud C, et al. Treatment in a specialised out-patient mood disorder clinic v. standard out-patient treatment in the early course of bipolar disorder: randomised clinical trial. Br J Psychiatry. 2013;202(3):212-9.

Marshall M, Rathbone J. Early intervention for psychosis. Schizophr Bull. 2011;37:1111-4.

Martin DJ, Smith DJ. Is there a clinical prodrome of bipolar disorder? A review of the evidence. Expert Rev Neurother. 2013;13(1):89-98.

Marwaha S, Thompson A, Upthegrove R, Broome MR. 15 Years on: early intervention for the new generation. Br J Psychiatry. 2016;209(3):186-8.

Marwaha S, Durrani A, Singh S. Employment outcomes in people with bipolar disorder: a systematic review. Acta Psychiatr Scand. 2013;128(3):179-93.

Matza LS, Rajagopalan KS, Thompson CL, de Lissovoy G. Misdiagnosed patients with bipolar disorder: comorbidities, treatment patterns, and direct treatment costs. J Clin Psychiatry. 2005;66(11):1432-40.

McGorry PD. Issues for DSM-V: clinical staging: a heuristic pathway to valid nosology and safer, more effective treatment in psychiatry. Am J Psychiatry. 2007;164(6):859.

Merikangas KR, Jin R, He JP, Kessler RC, Lee S, Sampson NA, et al. Prevalence and correlates of bipolar spectrum disorder in the world mental health survey initiative. Arch Gen Psychiatry. 2011;68(3):241-51.

Murru A, Primavera D, Oliva M, Meloni M, Vieta E, Carpiniello B. The role of comorbidities in duration of untreated illness for bipolar spectrum disorders. J Affect Disord. 2015;188:319-23.

Mwangi B, Wu MJ, Cao B, Passos IC, Lavagnino L, Keser Z, et al. Individualized prediction and clinical staging of bipolar disorders using neuroanatomical biomarkers. Biol Psychiatry Cogn Neurosci Neuroimaging. 2016;1:186-94.

Nery-Fernandes F, Quarantini LC, Guimarães JL, de Oliveira IR, Koenen KC, Kapczinski F, et al. Is there an association between suicide attempt and delay of initiation of mood stabilizers in bipolar I disorder? J Affect Disord. 2012;136(3):1082-7.

Pari A, Ahmed A, Simon J, Wolstenholme J, Geddes JR, Goodwin GM. Economic evaluations in bipolar disorder: a systematic review and critical appraisal. Bipolar Disord. 2014;16(6):557-82.
Patel R, Shetty H, Jackson R, Broadbent M, Stewart R, Boydell J, et al. Delays before diagnosis and initiation of treatment in patients presenting to mental health services with bipolar disorder. PLoS One. 2015;10(5):e0126530.

Post RM. Kindling and sensitization as models for affective episode recurrence, cyclicity, and tolerance phenomena. Neurosci Biobehav Rev. 2007;31(6):858-73.

Post RM, Altshuler LL, Frye MA, Suppes T, Keck PE Jr, McElroy SL, et al. Complexity of pharmacologic treatment required for sustained improvement in outpatients with bipolar disorder. J Clin Psychiatry. 2010;71(9):1176-86.

Rosa A, González-Ortega I, González-Pinto A, Echeburúa E, Comes M, MartínezÀran A, et al. One-year psychosocial functioning in patients in the early vs late stage of bipolar disorder. Acta Psychiatr Scand. 2012;125(4):335-41.

Salvadore G, Drevets WC, Henter ID, Zarate CA, Manji HK. Early intervention in bipolar disorder, part I: clinical and imaging findings. Early Interv Psychiatry. 2008;2(3):122-35.

Scott J, Colom F, Vieta E. A meta-analysis of relapse rates with adjunctive psychological therapies compared to usual psychiatric treatment for bipolar disorders. Int J Neuropsychopharmacol. 2007;10(01):123-9.

Swann AC, Bowden CL, Calabrese JR, Dilsaver SC, Morris DD. Differential effect of number of previous episodes of affective disorder on response to lithium or divalproex in acute mania. Am J Psychiatry. 1999;156(8):1264-6.

Tohen M, Vieta E, Gonzalez-Pinto A, Reed C, Lin D. Baseline characteristics and outcomes in patients with first episode or multiple episodes of acute mania. J Clin Psychiatry. 2010;71(3):255-61.

Torres I, DeFreitas C, DeFreitas V, Bond D, Kunz M, Honer W, et al. Relationship between cognitive functioning and 6-month clinical and functional outcome in patients with first manic episode bipolar I disorder. Psychol Med. 2011;41(05):971-82.

Wang PS, Berglund P, Olfson M, Pincus HA, Wells KB, Kessler RC. Failure and delay in initial treatment contact after first onset of mental disorders in the National Comorbidity Survey Replication. Arch Gen Psychiatry. 2005:62(6):603-13.

Whiteford HA, Degenhardt L, Rehm J, Baxter AJ, Ferrari AJ, Erskine HE, et al. Global burden of disease attributable to mental and substance use disorders: findings from the Global Burden of Disease Study 2010. Lancet. 2013;382(9904):1575-86.

\section{Submit your manuscript to a SpringerOpen ${ }^{\circ}$ journal and benefit from:}

- Convenient online submission

- Rigorous peer review

- Immediate publication on acceptance

- Open access: articles freely available online

- High visibility within the field

- Retaining the copyright to your article

Submit your next manuscript at springeropen.com 